\author{
Haben Sie Fragen zur Abrechnung oder zur wirtschaftlichen \\ Praxisführung? Als Leser der MMW können Sie sich an unseren \\ Experten wenden: Helmut Walbert, Facharzt für Allgemein- \\ medizin und Betriebswirt aus Würzburg.
}

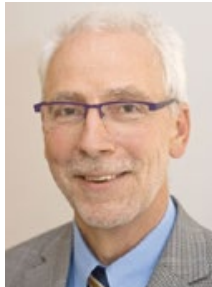

Helmut Walbert

Allgemeinarzt,

Medizinjournalist und

Betriebswirt Medizin

\title{
Vorhaltepauschale fällt bei Akupunktur weg
}

? Dr. S. F., Hausärztin, SchleswigHolstein: Ich erbringe in der Praxis Akupunkturleistungen. Jetzt habe ich gehört, dass dann die Zusatzpauscha-

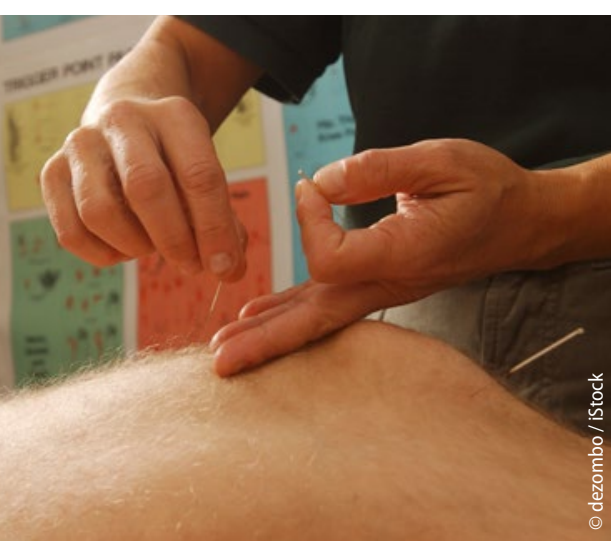

Das ist keine typische Hausarztleistung. le für die hausärztliche Grundversorgung gestrichen wird. Ist das richtig?

!

MMW-Experte Walbert: Die Nr. 03040, die sogenannte Vorhaltepauschale, ist vielen Hausärzten gar nicht bewusst, weil sie von der KV automatisch zugesetzt oder "gestrichen“ wird. Sie dient der Finanzierung der typischen Strukturen einer Hausarztpraxis zur Grundversorgung - man denke an die vielen nicht berechnungsfähigen Leistungen des Anhangs 1.

Um die Vorhaltepauschale zu erhalten, dürfen im Behandlungsfall allerdings keine Leistungen abgerechnet werden, die nicht grundsätzlich zum hausärztlichen Versorgungsauftrag gehören. Das schließt z.B. autogenes Training und Relaxationsbehandlung nach Jacobson (Nrn. 35111-35113) oder die Leistungen der Phlebologie aus dem EBMAbschnitt 30.5 aus - und ebenso die Schmerztherapie laut Abschnitt 30.7, wozu die Akupunktur zählt.

Die Vorhaltepauschale wird mit 15,03 Euro vergütet, die Akupunktur nach $\mathrm{Nr}$. 30791 mit 22,12 Euro. Man muss also nicht groß rechnen.

Kritischer wird es z. B. bei der Anleitung des Patienten zur Selbstanwendung der transkutanen elektrischen Nervenstimulation (TENS) nach Nr. 30712. Diese Leistung wird mit 6,99 Euro vergütet und kann im Behandlungsfall bis zu fünfmal angesetzt werden. Die wegfallende Vorhaltepauschale wird also ab der dritten Leistung ausgeglichen.

\section{Korrektur: Zwei persönliche Kontakte für Nr. 03221}

MMW-Experte Walbert: In MMW 11/2016 habe ich auf Seite 32 den Leistungsinhalt des kleinen Chronikerzuschlags nach Nr. 03221 falsch interpretiert. Ich hatte geschrieben, dass er auch dann angesetzt werden kann, wenn der zweite Kontakt ein telefonischer ist. Die Legende fordert allerdings mindestens zwei persönliche Arzt-PatientenKontakte, zu denen laut Punkt 4.3.1 der Allgemeinen Bestimmungen definitiv nicht der telefonische Kontakt zählt. Vielmehr müssen Arzt und Patient zur selben Zeit am selben Ort direkt interagieren.

Bei den Plausibilitätsprüfungen geraten die Chronikerziffern zurzeit in den Blickpunkt. Eine kurze Dokumentation ist also unabdingbar. Der Kommentar von Wezel/Liebold, die Beurteilungsgrundlage vieler KVen, fordert einen „bilateralen Informationsaustausch“, der diagnostischen oder therapeutischen Zielen dient und neben dem verbalen Austausch auch visuelle Elemente wie Gesten und Mimik umfasst.
Mit dieser Formulierung ist eindeutig der „großzügigen“ Interpretation widersprochen, dass die persönliche Überreichung eines Wiederholungsrezepts durch den Arzt in der Anmeldung oder ein freundliches „Hallo, wie geht's?“ bereits persönliche Arzt-Patienten-Kontakte darstellen.

Die Missachtung dieser Definitionen kann nicht nur zu Honorarrückforderungen führen, sondern auch den strafrechtlich relevanten Vorwurf des Abrechnungsbetruges bedeuten. 